\title{
High Clostridium difficile Infection among HIV-Infected Children with Diarrhea in a Tertiary Hospital in Mwanza, Tanzania
}

\author{
Mwanaisha Seugendo, ${ }^{1}$ Aldofine Hokororo, ${ }^{1}$ Rogatus Kabyemera, ${ }^{1}$ Delfina R. Msanga, \\ Mariam M. Mirambo, ${ }^{2}$ Vitus Silago, ${ }^{2}$ Uwe Groß, ${ }^{3}$ and Stephen E. Mshana $\mathbb{D}^{2}$ \\ ${ }^{1}$ Department of Pediatrics and Child Health, Weill Bugando School of Medicine, Mwanza, Tanzania \\ ${ }^{2}$ Department of Microbiology and Immunology, Weill Bugando School of Medicine, Mwanza, Tanzania \\ ${ }^{3}$ Institute of Medical Microbiology, University Medical Center Göttingen, Göttingen, Germany \\ Correspondence should be addressed to Stephen E. Mshana; stephen72mshana@gmail.com
}

Received 9 February 2020; Revised 23 July 2020; Accepted 10 August 2020; Published 28 August 2020

Academic Editor: Frederick J. Kaskel

Copyright (c) 2020 Mwanaisha Seugendo et al. This is an open access article distributed under the Creative Commons Attribution License, which permits unrestricted use, distribution, and reproduction in any medium, provided the original work is properly cited.

\begin{abstract}
Clostridium difficile causes a million of illnesses each year worldwide and can affect people of all ages. Limited data exist on the prevalence of $C$. difficile infections (CDI) among children below five years of age in developing countries. This study is aimed at determining the prevalence, associated factors, and outcome of the Clostridium difficile infection among children with diarrhea attending a tertiary hospital in Mwanza, Tanzania. Stool samples were collected and cultured anaerobically to isolate Clostridium difficile, followed by $C$. difficile toxin $\mathrm{A}$ and $\mathrm{B}$ assay and ribotyping. A total of 301 children with diarrhea were enrolled. A total of 22 (7.31\%, 95\% CI: 0.89-0.95) nonrepetitive stool samples were positive for Clostridium difficile. Eighteen (81\%) of C. difficile isolates were toxigenic, and $16(72.7 \%)$ had unknown ribotypes. Independent predictors of positive $C$. difficile were as follows: positive HIV status, hospital stay of more than four days, high stool leukocyte count, and watery stool. Clostridium difficile-positive children had significantly higher median duration of the diarrhea than those without $C$. difficile. Clinicians should consider $C$. difficile as a possible cause of diarrhea in children living in developing countries and institute appropriate management to prevent associated morbidities and mortalities. Furthermore, there is a need of joint effort to improve $C$. difficile diagnosis and surveillance in developing countries to establish the unknown epidemiology of $\mathrm{CDI}$ in these countries.
\end{abstract}

\section{Introduction}

C. difficile is a gram-positive, cytotoxin-producing anaerobic bacterium commonly existing in the large intestines of humans and animals without causing disease [1,2]. Clostridium difficile is one of the known causes of the antibioticassociated diarrhea [3, 4]. Worldwide, Clostridium difficile causes a million of illnesses each year and can affect people of all ages [5]. The incidence of Clostridium difficile infection (CDI) has rapidly increased since 1990, with a marked increase in mortality since 2000 [1].

There is little understanding of the epidemiology of CDI in children especially in the low- and middle-income countries (LMICs). Few studies exist in Africa regarding the magnitude of CDI; these studies have documented the prevalence to range from 4.9 to $8.6 \%$ [6-10]. Recently, the prevalence of CDI among adults and children with diarrhea in Mwanza has been documented to be 6.4\% [9]. Colonization of $C$. difficile among healthy children has been reported in a number of surveys and has been found to range from 2 to $64 \%$, with more colonization being reported in neonates and infants [11-13].

Use of antibiotics such as third-generation cephalosporins or ampicillin-sulbactam with an aminoglycoside has been documented to be strongly associated with CDI in children $[14,15]$. Other factors that have been found to be 
associated with CDI in children include the following: inflammatory bowel disease (IBD) [16] and use of proton pump inhibitors [17]. Few studies have reported CDI among HIV-infected children; a study from Poland [18] observed only 3 cases (17\%) of CDI among 18 HIV-infected children; in Tanzania, seven $(77.8 \%)$ out 9 C. difficile cases were children; and HIV infection was an independent factor that was associated with the occurrence of CDI. No study has been done to investigate the role of HAART and CDI in children; however, a multicenter study which included about $1 \%$ of children documented that CDI incidence was higher among untreated HIV-seropositive individuals but the incidence significantly declined during HAART treatment [19].

There is limited information regarding CDI in developing countries especially in children. The control of $C$. difficile requires understanding the magnitude and factors associated with CDI. A previous study [9], which included both adults and children, involved 108 children; the sample size was small to provide clear epidemiology of CDI in children in the study setting. Therefore, this study has documented the magnitude and factors associated with CDI among 301 children from a tertiary hospital in Mwanza, Tanzania.

\section{Material and Methods}

2.1. Study Design, Study Area, Inclusion Criteria, and Sampling. This was a hospital-based analytical crosssectional study with a follow-up component until diarrhea stopped. It was conducted at the Bugando Medical Centre (BMC), a tertiary hospital in Mwanza, Tanzania, between February and September 2016.

All children aged 2 months to 12 years admitted with diarrhea (regardless of duration of the diarrhea) during the study period were serially enrolled until the desired sample size was reached. All inpatients who developed diarrhea while in the ward were also eligible to be enrolled. Demographic and clinical variables were collected using a standardized data collection tool. Diarrhea was defined as per World Health Organization "Diarrheal disease. 2013” [20].

2.2. Laboratory Procedures. All laboratory investigations were done following recommended SOPs as per the Bugando Medical Centre accredited laboratory ISO15189 by Southern African Development Community Accreditation Service (SADCAS) accreditation body with registration number MD 002. Nonrepetitive stool samples from children were collected and taken to the laboratory within 2 hours of collection. The watery/mucoid part of stool samples was cultured on selective Clostridium agar (BioMérieux, Paris, France) and incubated in the anaerobic condition (Anaerobic Pack BioMérieux, Marcy l'Etoile, France) for 24 hours as previously described [21].

Presumptive colonies of $C$. difficile were tested for glutamate dehydrogenase and $C$. difficile toxins $\mathrm{A}$ and $\mathrm{B}$ using rapid commercial tests (Quik Chek Complete, Alere TechLab, Blacksburg, VA, USA). Furthermore, multiplex PCR was done to detect toxin gene profiles [22] and PCR ribotyped by agarose or capillary gel electrophoresis was done to establish ribotypes as previously described [23]. In addi- tion, stool was examined for leukocytes as previously described [24].

HIV testing was done according to the Tanzania national algorithm [25] using the SD Bioline HIV rapid test (Standard Diagnostic Inc., Korea) as the first test followed by Uni-Gold (Trinity Biotech, Ireland) as the second test. For children below 18 months, PCR was used to confirm HIV status as per National AIDS Control Programme (NACP) recommendations [25].

All patients enrolled in the study were assessed and managed as per BMC treatment guidelines as well as per WHO protocols. The antibiotic treatment in the pediatrics department includes ampicillin and gentamicin as the first line and third-generation cephalosporin as the second line. All children who were $C$. difficile positive were treated by metronidazole as recommended by South Africa treatment guidelines for CDI [26].

Children were followed, and the duration taken for when diarrhea stopped was recorded.

2.3. Data Analysis. Data were entered in the computer using Excel 2007 and cleaned and analyzed using STATA version 13 (College Station, Texas). Data were summarized in frequency tables and bar charts. Continuous variables (age and duration of when diarrhea stopped) were summarized as medians, while categorical variables were summarized as proportions. In this study, we defined C. difficile infection (CDI) as the acute onset of diarrhea with documented toxigenic C. difficile or its toxin or the isolation of $C$. difficile from the child with clinical manifestation [27]. To determine factors associated with $C$. difficile, backward univariate followed by multivariate logistic regression analyses were performed. All factors with $P$ value less than 0.05 on univariate analysis were subjected on the multivariate logistic regression analysis. Cox regression analyses with Kaplan-Meier curves were used to assess the hazard ratio in relation to the outcome (diarrhea stopped). The 95\% confidence interval was determined, and predictors with $P$ value of less than 0.05 were considered to be statistically significant.

2.4. Ethical Considerations. The protocol of this study was approved by the Joint CUHAS-Bugando Ethics and Scientific Review Committee. Written Informed consent for the participation in the study was obtained from the mother/guardians of the respective child.

\section{Results}

3.1. Sociodemographic and Clinical Features. The median age of participants was 12 [IQR: 6-24] months, and approximately $156(51.8 \%)$ of the children were males, and 164 (54.5\%) of the enrolled children were below 12 months. Among 301 children enrolled into this study, 266 (88.4\%) had history of using antibiotics before enrollment. The most frequently used antibiotics were as follows: amoxicillin, ampicillin, trimethoprim/sulfamethoxazole, erythromycin, and metronidazole. A total of 33 (11\%) children were HIV positive (Table 1). 
TABle 1: Sociodemographic and clinical characteristics of 301 children with diarrhea.

\begin{tabular}{|c|c|c|}
\hline Study variable & Number of patients & Percent \\
\hline \multicolumn{3}{|l|}{ Age in months } \\
\hline$\leq 12$ months & 164 & 54.5 \\
\hline$>12$ months & 137 & 45.5 \\
\hline \multicolumn{3}{|l|}{ Sex } \\
\hline Male & 156 & 51.8 \\
\hline Female & 145 & 48.2 \\
\hline \multicolumn{3}{|l|}{ Type of stool } \\
\hline Watery & 151 & 50.2 \\
\hline Loose pasty & 150 & 49.8 \\
\hline \multicolumn{3}{|l|}{ Residence } \\
\hline Urban & 194 & 64.5 \\
\hline Rural & 107 & 35.5 \\
\hline \multicolumn{3}{|l|}{ HIV status } \\
\hline Positive & 33 & 10.96 \\
\hline Negative & 268 & 89.04 \\
\hline \multicolumn{3}{|l|}{ Antibiotic use } \\
\hline Yes & 266 & 88.4 \\
\hline No & 35 & 11.6 \\
\hline \multicolumn{3}{|c|}{ Type of toilet use } \\
\hline Flush & 115 & 38.2 \\
\hline Pit & 186 & 61.8 \\
\hline \multicolumn{3}{|c|}{ Type of water source } \\
\hline Tap & 205 & 68.1 \\
\hline Nontap & 96 & 31.9 \\
\hline \multicolumn{3}{|c|}{ Dehydration status } \\
\hline No & 158 & 52.5 \\
\hline Some & 111 & 36.9 \\
\hline Severe & 32 & 10.6 \\
\hline \multicolumn{3}{|l|}{ Parental income } \\
\hline $0-500 \mathrm{~K}$ & 223 & 74.09 \\
\hline $500 \mathrm{~K}-1 \mathrm{M}$ & 60 & 19.93 \\
\hline$>1 \mathrm{M}$ & 18 & 5.98 \\
\hline \multicolumn{3}{|l|}{ Herbal use } \\
\hline Yes & 176 & 58.5 \\
\hline No & 125 & 41.5 \\
\hline
\end{tabular}

3.2. Prevalence and Factors Associated with C. difficile. Out of 301 children with diarrhea, 22 (7.3\%, 95\% CI: 4.4-10.2) had positivite $C$. difficile in their stool, hence possible CDI. All infected children were below 5 years of age. Other pathogenic bacteria detected from the stool were Salmonella spp. (19, $5.7 \%)$ and Shigella spp. (6, 1.9\%). No patient had coinfection with $C$. difficile.

Out of 151 participants who presented with watery stool, 21 (13.9\%) were C. difficile positive compared to $0.7 \%$ of 150 participants with loose pasty stool $(P=0.002)$. HIV-positive children had significantly more often $C$. difficile-positive stool samples than HIV-negative children (24.2\% vs. $5.2 \%$, $P<0.001)$. We also identified $C$. difficile significantly more often in patients who had a prolonged median hospital stay before diarrhea started than in those with a shorter median hospital stay (4.5 [4-8] days vs. 3 [1-7] days, $P=0.026)$. Clostridium difficile-positive children had significantly higher median duration (days) of diarrhea than those without $C$. difficile (6 [4-10] vs. 3 [2-5], $P=0.001$ ).

Factors that independently predicted CDI were as follows: being HIV positive (OR: 7.9, 95\% CI: 1.86-33.5, $P=$ 0.005 ), watery stool (OR: $21.4,95 \% \mathrm{CI}: 1.3-343.8, P=$ 0.003 ), median hospital stay before diarrhea (OR: 1.12, 95\% CI: 1.01-1.23, $P=0.026)$, median stool leukocytes (OR: 1.05, 95\% CI: 1.02-1.23, $P<0.001$ ), and median duration of diarrhea (OR: 1.16, 95\% CI: 1.05-1.3, $P=0.003$ ) (Table 2).

3.3. C. difficile Ribotypes, Toxin A, and Toxin B in 13 Strains That Were Typed. The majority $(9 / 13(69 \%))$ of C. difficile isolates had unknown ribotypes. Toxin A and toxin B were detected in $9 / 13$ of $C$. difficile isolates typed. Ribotypes 38 and 84 were detected in one isolate each and ribotype 45 in 2 isolates (Table 3 ).

3.4. Outcome in relation to When Diarrhea Stopped. The outcome of CDI as measured by duration of when diarrhea stopped was determined. Children with CDI had significantly higher median duration of diarrhea than those without CDI (6 [4-10] days vs. 2 [2-4] days, $P \leq 0.001$ ) (Figure 1). Based on Cox regression analysis, the rate of stopping diarrhea was significantly fast in children without CDI (HR: 0.4, 95\% CI: 0.2-0.6, $P<0.001$ ) (Figure 2).

\section{Discussion and Conclusion}

This study has observed the prevalence of CDI to be $7.3 \%$ among children with diarrhea which is comparable with previous studies in Tanzania (6.4\%), Zimbabwe (8.6\%), South Africa (7.1\%), and Brazil (6.7\%) [6, 7, 9, 28]. Comparing with other studies outside Africa (India (15\%) and South Korea $(13.2 \%))[29,30]$, the observed prevalence in the current study is significantly low which could be explained by fact that the studies with high prevalence used enzyme immunoassay (EIA) to detect cytotoxin; EIAs have been found to be more sensitive than standard culture technique and rapid toxin assays which were used in the current study [27]. Generally, in developed countries, among hospitalized children, the incidence of CDI follows an increasing trend necessitating more studies in developing countries to monitor the trend of CDI [31].

No coinfection between $C$. difficile and other organisms was observed in the current study which is different from other studies in South Africa, India, the United Kingdom, and Germany where coinfection with Shigella spp. and Salmonella spp. was linked to severe disease, high chances of complications, and reoccurrences [2, 7, 29, 32, 33].

In this study, circulating ribotypes included 038, 045, and 084 with majority of unknown ribotypes observed in our patients. The ribotype 084 has been recently found to be the predominant ribotype in Ghana $[8,10]$. Most of the isolates typed in this study were toxigenic cementing their role 
TABLE 2: Factors associated with CDI among study population $(n=301)$.

\begin{tabular}{|c|c|c|c|c|c|c|}
\hline \multirow{2}{*}{ Variable } & \multicolumn{2}{|c|}{ C. difficile infection } & \multicolumn{2}{|c|}{ Univariate } & \multicolumn{2}{|c|}{ Multivariate } \\
\hline & Yes $(\%)$ & No (\%) & OR [95\% CI] & $P$ value & OR $[95 \% \mathrm{CI}]$ & $P$ value \\
\hline \multicolumn{7}{|l|}{ Sex } \\
\hline Male & $12(7.7)$ & $144(92.3)$ & 1.0 & & & \\
\hline Female & $10(6.9)$ & $135(93.1)$ & $0.9[0.4-2.1]$ & 0.791 & & \\
\hline \multicolumn{7}{|l|}{ Age in months } \\
\hline$\leq 12$ months & $9(5.5)$ & $155(94.5)$ & 1.0 & & & \\
\hline$>12$ months & $13(9.5)$ & $124(90.5)$ & $1.8[0.7-4.3]$ & 0.189 & & \\
\hline \multicolumn{7}{|l|}{ Residence } \\
\hline Urban & $13(6.7)$ & $181(93.3)$ & 1.0 & & & \\
\hline Rural & $9(8.4)$ & $98(91.6)$ & $1.3[0.5-3.1]$ & 0.586 & & \\
\hline \multicolumn{7}{|l|}{ Antibiotic use } \\
\hline No & $1(2.9)$ & $34(97.1)$ & 1.0 & & & \\
\hline Yes & $21(7.9)$ & $245(92.1)$ & $2.9[0.4-22.4]$ & 0.304 & & \\
\hline \multicolumn{7}{|l|}{ HIV status } \\
\hline Negative & $14(5.2)$ & $254(94.8)$ & 1.0 & & & \\
\hline Positive & $8(24.2)$ & $25(75.8)$ & $5.8[2.2-15.2]$ & $<0.001$ & $7.9[1.86-33.5]$ & 0.005 \\
\hline Median diarrhea frequency (days) & $9[8-10]$ & 4 [3-7] & $1.4[1.2-1.5]$ & $<0.001$ & $1.04[0.82-1.32]$ & 0.753 \\
\hline \multicolumn{7}{|l|}{ Type of stool } \\
\hline Loose pasty & $1(0.7)$ & $149(99.3)$ & 1.0 & 0.002 & \multirow{2}{*}{$21.4[1.3-343.9]$} & 0.003 \\
\hline Watery & $21(13.9)$ & $130(86.1)$ & $24.1[3.2-181.1]$ & & & \\
\hline \multicolumn{7}{|l|}{ Comorbidities } \\
\hline No & $7(7.7)$ & $84(92.3)$ & 1.0 & & & \\
\hline Yes & $15(7.1)$ & $195(92.9)$ & $0.9[0.4-2.3]$ & 0.866 & & \\
\hline Median hospital stay before diarrhea (days) & $4.5[4-8]$ & $3[1-7]$ & $1.1[1.01-1.14]$ & 0.024 & $1.12[1.01-1.23]$ & 0.026 \\
\hline Median body temperature & $38.2[37.9-39]$ & $37[36.0-37.5]$ & $2.8[1.8-4.2]$ & $<0.001$ & $1.4[0.9-2.5]$ & 0.173 \\
\hline Median stool leukocytes & $55[45-70]$ & $0[0-4]$ & $1.06[1.04-1.08]$ & $<0.001$ & $1.05[1.02-0.07]$ & $<0.001$ \\
\hline Median diarrhea duration & $6[4-10]$ & $3[2-5]$ & $1.11[1.04-1.18]$ & 0.001 & $1.16[1.05-1.30]$ & 0.003 \\
\hline
\end{tabular}

Table 3: Characteristic of C. difficile ribotypes $(n=13)$.

\begin{tabular}{lccccc}
\hline SNO & Strain & Age (months) & Ribotype & ToxA & ToxB \\
\hline 1 & 017 & 11 & Unknown & NEG & NEG \\
2 & O73 & 8 & 084 & NEG & NEG \\
3 & 100 & 6 & Unknown & POS & POS \\
4 & 171 & 60 & Unknown & POS & POS \\
5 & 210 & 11 & Unknown & NEG & NEG \\
6 & 221 & 24 & Unknown & POS & POS \\
7 & 268 & 8 & Unknown & POS & POS \\
8 & 270 & 21 & Unknown & POS & POS \\
9 & 033 & 12 & Unknown & POS & POS \\
10 & 091 & 15 & Unknown & POS & POS \\
11 & 018 & 2 & 038 & NEG & NEG \\
12 & 015 & 60 & 045 & POS & POS \\
13 & 022 & 13 & 045 & POS & POS \\
\hline
\end{tabular}

in causing diarrhea in symptomatic children as previously documented [34].

In this study, we assessed the association between comorbidities and CDI [35]; we confirmed that positive HIV status was significantly associated with CDI similar to the findings from the previous study which involved both adults and children [9]. A study in South Africa did not find the association between CDI and HIV infection, and this was due to the fact that the HIV status was not known in a significant proportion of participants resulting in a low number of HIVpositive individuals in the subanalysis [7]. The findings regarding the association between HIV and CDI will help clinicians to have a high index of suspicion of CDI among $\mathrm{HIV}$-infected individuals with history of hospitalization and antibiotic use. In addition, clinicians should also note that $\mathrm{HIV}$-infected individuals are prone to opportunistic infections (OIs) requiring these patients to have frequent or prolonged courses of antimicrobial therapy contributing to the risk of CDI. Furthermore, studies have clearly shown the decrease in the incidence of CDI with HAART use [36].

Hospital stay for more than 5 days was significantly associated with CDI; similar observations were made by studies from India and Malaysia, which documented that patients who stayed in hospital settings for longer duration were more likely to acquire CDI $[29,37]$. It was further observed that children with CDI significantly had longer hospital stay than those with no CDI $[3,35,38]$. CDI has been found to be associated with the local inflammatory process and systemic 


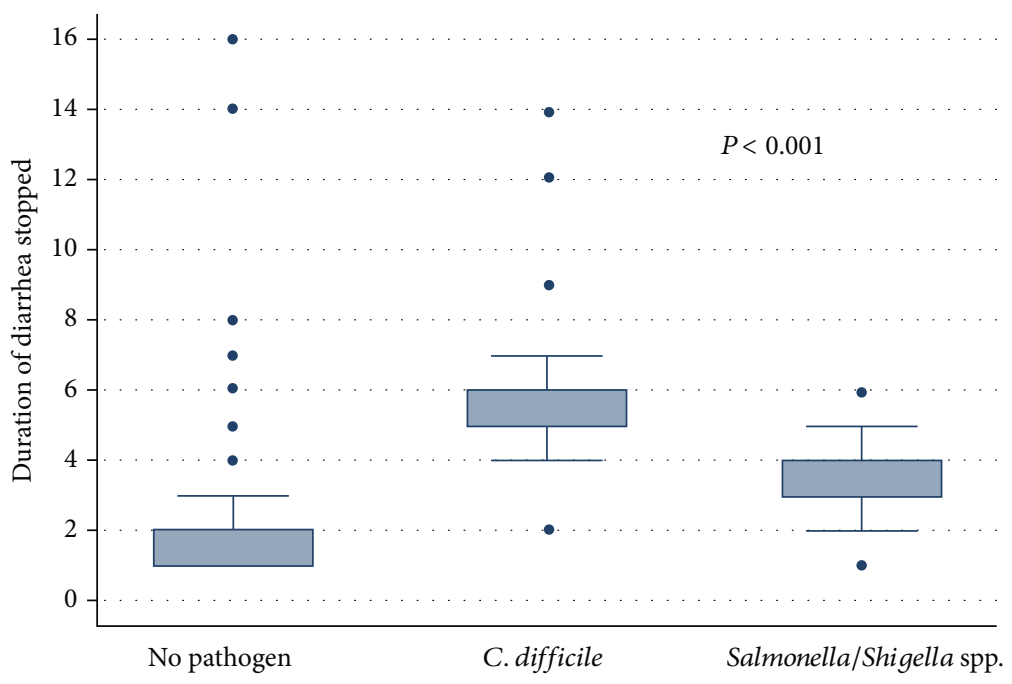

FIGURE 1: Median duration of when diarrhea stopped in 301 children (no pathogen, C. difficile, and Salmonella spp./Shigella spp.).

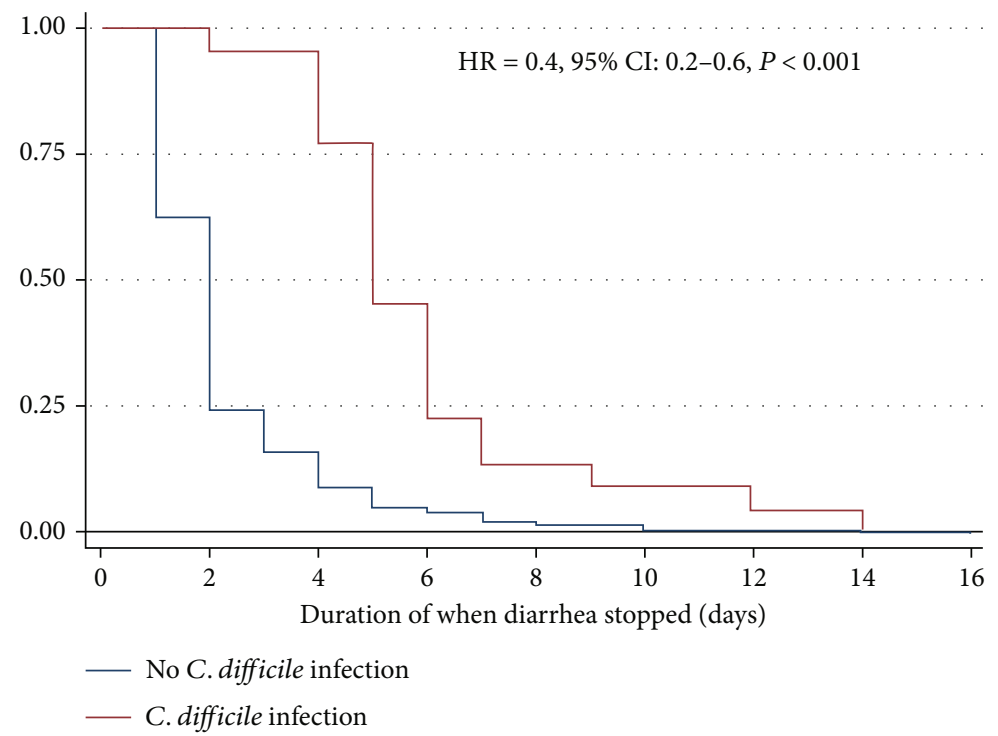

FIGURE 2: Kaplan-Meier curve showing the rate of when diarrhea stopped between CDI positive and CDI negative.

response [39], hence serious disease. This was confirmed in the current study whereby children with CDI had significantly more leukocytes in stool than those without CDI. Clostridium-positive children had significant longer duration of diarrhea than those without $C$. difficile and those infected with other organisms. This was also observed in a study in Poland [35] which showed a minimum of 3-4 days of recovery after starting treatment of CDI, but few cases had prolonged diarrhea of up to 6 days. In the USA [40], children with CDI had median hospital stay of 5.5 days similar to what has been observed in the present study. Another study in the USA observed increased pediatric CDI-related hospitalizations [41].

In the present study, in contrast to previous studies which showed a significant association between CDI and antibiotic use $[9,16,27]$, a nonsignificant association was observed in the current study. This could be explained by the fact that the majority $(88 \%)$ of children had history of using antibi- otics providing unequal distribution of the outcome in different groups. We observed only one child to be CDI positive among 35 children with no history of antibiotic use.

One of the limitations of this study is the lack of a comparative group because asymptomatic infection has been observed in children $[31,42]$; however, the study has provided information regarding CDI in these children with diarrhea and associated factors. It should be noted that to mitigate this limitation, the study excluded children below 2 months who are prone to be colonized with $C$. difficile $[34,43]$.

\section{Conclusion}

Toxigenic Clostridium difficile infections are prevalent among children below five years of age with diarrhea, and these infections are independently predicted by positive HIV status, prolonged hospital stay of more than four days, 
high leukocytes in stool, and watery stool. In addition, CDI was found to be associated with prolonged duration of diarrhea. Clinicians should consider CDI in children with diarrhea and initiate appropriate management to prevent associated morbidities and mortalities. C. difficile diagnosis and surveillance is urgently needed in developing countries to establish the unknown epidemiology of CDI in these countries.

\section{Data Availability}

The majority of data have been included in the manuscript; other data used to support the findings of this study are available from the corresponding author upon request.

\section{Conflicts of Interest}

The authors declare that they have no competing interests.

\section{Acknowledgments}

We are grateful to the parents who allowed their children to participate in the study. We acknowledge the determination of toxin genes and ribotypes by the German Consulting Laboratory for Clostridium difficile and the assistance and guidance provided by all staff members of the Department of Pediatrics, Bugando Medical Center, as well as the Department of Microbiology/Immunology, Catholic University of Health and Allied Sciences, Bugando Hospital, and Institute of Medical Microbiology Göttingen. This work was supported by the Catholic University of Health and Allied Sciences and Institute of Medical Microbiology Göttingen.

\section{References}

[1] M. Rupnik, M. H. Wilcox, and D. N. Gerding, "Clostridium difficile infection: new developments in epidemiology and pathogenesis," Nature Reviews Microbiology, vol. 7, no. 7, pp. 526-536, 2009.

[2] S. Pai, S. H. Aliyu, D. A. Enoch, and J. A. Karas, "Five years experience of Clostridium difficile infection in children at a UK tertiary hospital: proposed criteria for diagnosis and management," PLoS One, vol. 7, no. 12, article e51728, 2012.

[3] J. Y. Na, J. M. Park, K. S. Lee, J. O. Kang, S. H. Oh, and Y. J. Kim, "Clinical characteristics of symptomatic Clostridium difficile infection in children: conditions as infection risks and whether probiotics is effective," Pediatric Gastroenterology, Hepatology \& Nutrition, vol. 17, no. 4, pp. 232-238, 2014.

[4] N. Rajabally, M. Pentecost, G. Pretorius, A. Whitelaw, M. Mendelson, and G. Watermeyer, "The Clostridium difficile problem: a South African tertiary institution's prospective perspective," South African Medical Journal, vol. 103, no. 3, pp. 168-172, 2013.

[5] K. E. Burke and J. T. Lamont, "Clostridium difficile infection: a worldwide disease," Gut and Liver, vol. 8, no. 1, pp. 1-6, 2014.

[6] C. Simango and S. Uladi, "Detection of Clostridium difficile diarrhoea in Harare, Zimbabwe," Transactions of the Royal Society of Tropical Medicine and Hygiene, vol. 108, no. 6, pp. 354-357, 2014.
[7] A. Samie, C. L. Obi, J. Franasiak et al., "PCR Detection of Clostridium difficile Triose Phosphate Isomerase (tpi), Toxin A (tcdA), Toxin B (tcdB), Binary Toxin (cdtA, cdtB), and tcdC Genes in Vhembe District, South Africa," The American Journal of Tropical Medicine and Hygiene, vol. 78, no. 4, pp. 577585, 2008.

[8] I. Janssen, P. Cooper, K. Gunka et al., "High prevalence of nontoxigenic Clostridium difficile isolated from hospitalized and non-hospitalized individuals in rural Ghana," International Journal of Medical Microbiology, vol. 306, no. 8, pp. 652-656, 2016.

[9] M. Seugendo, S. E. Mshana, A. Hokororo et al., "Clostridium difficile infections among adults and children in Mwanza/Tanzania: is it an underappreciated pathogen among immunocompromised patients in sub-Saharan Africa?," New Microbes and New Infections, vol. 8, pp. 99-102, 2015.

[10] M. Seugendo, I. Janssen, V. Lang et al., "Prevalence and strain characterization of Clostridioides (Clostridium) difficile in representative regions of Germany, Ghana, Tanzania and Indonesia-a comparative multi-center cross-sectional study," Frontiers in Microbiology, vol. 9, article 1843, 2018.

[11] E. Holst, I. Helin, and P.-A. Mårdh, "Recovery of Clostridium difficile from children," Scandinavian Journal of Infectious Diseases, vol. 13, no. 1, pp. 41-45, 1981.

[12] H. E. Larson, F. E. Barclay, P. Honour, and I. D. Hill, "Epidemiology of Clostridium difficile in infants," The Journal of Infectious Diseases, vol. 146, no. 6, pp. 727-733, 1982.

[13] R. P. Bolton, S. K. Tait, P. R. Dear, and M. S. Losowsky, "Asymptomatic neonatal colonisation by Clostridium difficile," Archives of Disease in Childhood, vol. 59, no. 5, pp. 466-472, 1984.

[14] S. Tschudin-Sutter, P. D. Tamma, A. M. Milstone, and T. M. Perl, "Predictors of first recurrence of Clostridium difficile infections in children," The Pediatric Infectious Disease Journal, vol. 33, no. 4, pp. 414-416, 2014.

[15] F. Oĝuz, G. Uysal, S. Daşdemir, H. Oskovi, and S. Vidinlisan, "The role of Clostridium difficile in childhood nosocomial diarrhea," Scandinavian Journal of Infectious Diseases, vol. 33, no. 10, pp. 731-733, 2001.

[16] F. Pascarella, M. Martinelli, E. Miele, M. Del Pezzo, E. Roscetto, and A. Staiano, "Impact of Clostridium difficile infection on pediatric inflammatory bowel disease," The Journal of Pediatrics, vol. 154, no. 6, pp. 854-858, 2009.

[17] R. Turco, M. Martinelli, E. Miele et al., "Proton pump inhibitors as a risk factor for paediatric Clostridium difficile infection," Alimentary Pharmacology \& Therapeutics, vol. 31, no. 7, pp. 754-759, 2010.

[18] G. Martirosian, J. Popielska, and M. Marczyńska, “Occurrence of Clostridium difficile in fecal samples of HIVinfected children in Poland," Anaerobe, vol. 9, no. 6, pp. 295-297, 2003.

[19] T. H. Sanchez, J. T. Brooks, P. S. Sullivan et al., "Bacterial diarrhea in persons with HIV infection, United States, 1992-2002," Clinical Infectious Diseases, vol. 41, no. 11, pp. 1621-1627, 2005.

[20] World Health Organization (WHO), Diarrhoeal disease 2013, 2015, Reference Source.

[21] L. M. Marler, J. A. Siders, L. C. Wolters, Y. Pettigrew, B. L. Skitt, and S. D. Allen, "Comparison of five cultural procedures for isolation of Clostridium difficile from stools," Journal of Clinical Microbiology, vol. 30, no. 2, pp. 514-516, 1992. 
[22] J. Stahlmann, M. Schönberg, M. Herrmann, and L. von Müller, "Detection of nosocomial Clostridium difficile infections with toxigenic strains despite negative toxin A and B testing on stool samples," Clinical Microbiology and Infection, vol. 20, no. 9, pp. O590-O592, 2014.

[23] S. Janezic and M. Rupnik, "Molecular typing methods for Clostridium difficile: pulsed-field gel electrophoresis and PCR ribotyping," in Clostridium difficile, P. Mullany and A. P. Roberts, Eds., vol. 646 of Methods in Molecular Biology ${ }^{\mathrm{TM}}$, , pp. 55-65, Humana, 2010.

[24] B. J. Stoll, R. I. Glass, H. Banu, M. I. Huq, M. Khan, and M. Ahmed, "Value of stool examination in patients with diarrhoea," BMJ, vol. 286, no. 6383, pp. 2037-2040, 1983.

[25] E. F. Lyamuya, S. Aboud, W. K. Urassa et al., "Evaluation of simple rapid HIV assays and development of national rapid HIV test algorithms in Dar es Salaam, Tanzania," BMC Infectious Diseases, vol. 9, no. 1, p. 19, 2009.

[26] L. Legenza, S. Barnett, W. Rose, M. Bianchini, N. Safdar, and R. Coetzee, "Epidemiology and outcomes ofClostridium difficileinfection among hospitalised patients: results of a multicentre retrospective study in South Africa," BMJ Global Health, vol. 3, no. 4, article e000889, 2018.

[27] J. O. Shim, "Clostridium difficile in children: to treat or not to treat?," Pediatric Gastroenterology, Hepatology \& Nutrition, vol. 17, no. 2, pp. 80-84, 2014.

[28] L. J. F. Pinto, A. P. P. Alcides, E. O. Ferreira et al., "Incidence and importance of Clostridium difficile in paediatric diarrhoea in Brazil," Journal of Medical Microbiology, vol. 52, no. 12, pp. 1095-1099, 2003.

[29] S. Justin, B. Antony, K. V. Shenoy, and R. Boloor, "Prevalence of Clostridium difficile among paediatric patients in a tertiary care hospital, coastal Karnataka, South India," Journal of Clinical and Diagnostic Research, vol. 9, no. 2, pp. DC04-DC07, 2015.

[30] B.-M. Shin and E.-Y. Kuak, "Characterization of a toxin Anegative, toxin B-positive variant strain of Clostridium difficile," The Korean Journal of Laboratory Medicine, vol. 26, no. 1, pp. 27-31, 2006.

[31] A. Deshpande, C. Pant, M. P. Anderson, C. J. Donskey, and T. J. Sferra, "Clostridium difficile infection in the hospitalized pediatric population: increasing trend in disease incidence," The Pediatric Infectious Disease Journal, vol. 32, no. 10, pp. 1138-1140, 2013.

[32] G. Ackermann, S. Thomalla, F. Ackermann, R. Schaumann, A. C. Rodloff, and B. R. Ruf, "Prevalence and characteristics of bacteria and host factors in an outbreak situation of antibiotic-associated diarrhoea," Journal of Medical Microbiology, vol. 54, no. 2, pp. 149-153, 2005.

[33] R. E. El Feghaly, J. L. Stauber, P. I. Tarr, and D. B. Haslam, "Viral co-infections are common and are associated with higher bacterial burden in children with Clostridium difficile infection," Journal of Pediatric Gastroenterology and Nutrition, vol. 57, no. 6, pp. 813-816, 2013.

[34] J. Kim, S. A. Smathers, P. Prasad, K. H. Leckerman, S. Coffin, and T. Zaoutis, "Epidemiological features of Clostridium difficile-associated disease among inpatients at children's hospitals in the United States, 2001-2006," Pediatrics, vol. 122, no. 6, pp. 1266-1270, 2008.

[35] K. Dulęba, M. Pawłowska, and M. Wietlicka-Piszcz, "Clostridium difficile infection in children hospitalized due to diarrhea," European Journal of Clinical Microbiology \& Infectious Diseases, vol. 33, no. 2, pp. 201-209, 2014.
[36] P. J. Collini, E. Kuijper, and D. H. Dockrell, "Clostridium difficile infection in patients with HIV/AIDS," Current HIV/AIDS Reports, vol. 10, no. 3, pp. 273-282, 2013.

[37] S. Asma'Hassan, F. M. I. MPath, Z. A. R. MPath et al., "Prevalence of Clostridium difficile toxin in diarhoeal stool samples of patients from a tertiary hospital in North Eastern Penisular Malaysia," The Medical Journal of Malaysia, vol. 67, no. 4, p. 403, 2012.

[38] K. L. Schwartz, I. Darwish, S. E. Richardson, M. R. Mulvey, and N. Thampi, "Severe clinical outcome is uncommon in Clostridium difficile infection in children: a retrospective cohort study," BMC Pediatrics, vol. 14, no. 1, p. 28, 2014.

[39] V. Viswanathan, M. Mallozzi, and G. Vedantam, "Clostridium difficile infection: an overview of the disease and its pathogenesis, epidemiology and interventions," Gut Microbes, vol. 1, no. 4, pp. 234-242, 2010.

[40] J. S. Sammons, R. Localio, R. Xiao, S. E. Coffin, and T. Zaoutis, "Clostridium difficile infection is associated with increased risk of death and prolonged hospitalization in children," Clinical Infectious Diseases, vol. 57, no. 1, pp. 1-8, 2013.

[41] M. D. Zilberberg, G. S. Tillotson, and L. C. McDonald, “Clostridium difficile infections among hospitalized children, United States, 1997-2006," Emerging Infectious Diseases, vol. 16, no. 4, pp. 604-609, 2010.

[42] S. Jangi and J. T. Lamont, "Asymptomatic colonization by Clostridium difficile in infants: implications for disease in later life," Journal of Pediatric Gastroenterology and Nutrition, vol. 51, no. 1, pp. 2-7, 2010.

[43] S. Richardson, P. Alcock, and J. Gray, "Clostridium difficile and its toxin in healthy neonates," British Medical Journal, vol. 287 , no. 6396, p. 878, 1983. 